\title{
Rates of death and euthanasia for cats in no-kill shelters in the Czech Republic
}

\author{
Vladimír Večerek, Kateřina Kubesová, Eva Voslářová, Iveta Bedáňová \\ University of Veterinary and Pharmaceutical Sciences Brno, Faculty of Veterinary Hygiene and Ecology, \\ Department of Animal Protection, Welfare and Behaviour, Brno, Czech Republic
}

Received September 1, 2016

Accepted February 17, 2017

\begin{abstract}
Successful reduction of death and euthanasia rates requires the determination of factors related to death and euthanasia of cats in shelters. In the period from 2011 to 2015, 474 cats died and 240 had to be euthanized for health reasons in the three cat shelters monitored in our study. Altogether, death was the ultimate outcome for $33 \%$ of the cats admitted in the shelters. Out of the cats that died, $67.3 \%$ cats died within the first month and $95.4 \%$ within 6 months of admission to the shelter. Similarly, $65.0 \%$ of all euthanized cats were euthanized within the first month and $88.3 \%$ were euthanized within 6 months of admission. Since the majority of mortalities occurred shortly after admission, they more probably resulted from the cats' poor condition at the time of admission rather than from the shelter's environment. Females and cats younger than 6 months were at an increased risk of mortality. Autumn and summer were the seasons with the majority of fatalities. In general, the number of cats that died was almost double the number of cats that were euthanized; this suggests that attempts to cure cats were favoured over euthanasia, even in cases of serious health problems. In the fields of cat welfare and veterinary healthcare, the results enable increased attention to be focused on the determined risk factors and thus are an important prerequisite for a successful solution to the problem of the high number of deaths and instances of euthanasia of cats in shelters.
\end{abstract}

Feline, sanctuary, mortality, season, age

Millions of cats and dogs are annually impounded in animal shelters worldwide and the main fate of these captured animals is euthanasia. The number of dogs and cats annually euthanized in the United States has been estimated at between 5 million and 17 million (Carter 1990; Rowan 1992; Arkow 1994; Patronek et al. 1996). Other authors have estimated that between one tenth and one quarter of the companion animal population is destroyed each year, making euthanasia the leading cause of death in companion animals (Nassar et al. 1984). In 2003, 76,321 (57\%) cats were euthanized in Michigan shelters alone with the estimated ratio of euthanized cats to cats who had owners to be $3.1 \%$ (Bartlett et al. 2005). Also in Australia, more than half of the stray and unwanted cats admitted to animal shelters are euthanized (Alberthsen et al. 2013). In Japan, 93\% out of 164,308 cats admitted to animal shelters were euthanized in 2010; the number remaining largely unchanged for a long time (Onodera et al. 2014). The majority of shelter cats are euthanized due to overcrowding. The management of unwanted cats, in particular the large-scale euthanasia of often healthy cats, raises ethical issues (Rogelberg et al. 2007; Baran et al. 2009) and is of increasing concern to the community.

In some countries, laws prohibiting euthanasia of healthy animals are in force. In 2008, the State of São Paulo, Brazil, approved a law that regulates the use of the euthanasia of captured dogs and cats. With this new legislation, culling is no longer considered an acceptable control strategy, but reserved for reduction of suffering and the removal of aggressive animals, leaving only sterilization and adoption as remaining strategies for stray animal control (Ardila Galvis et al. 2015). However, even in this case the main fate of 
captured animals (cats and dogs) kept in the zoonosis control centres in Votorantim was euthanasia (44.3\%) and adoption the second (30.4\%). Among euthanized animals, many were killed on the first day of capture because they were in a condition of extreme suffering (due to fights, disease, been run over by a car, or for other health-related reasons). The other most frequent fate of the animals captured was death due to injury or disease. Infectious diseases are a major problem within many cat shelters (Cave et al. 2002). As several studies have indicated, the likelihood that a cat will contract an infection is significantly impacted by their length of time in a shelter (Pedersen et al. 2004; Dinnage et al. 2009). However, a study analyzing the risk of mortality in cats kept at the adoption centres of a UK cat charity revealed that the risk of mortality decreased as the time in the adoption centre increased (Murray et al. 2008). Based on retrospective data collected for 194 cases (cats that died or were euthanized) and 320 controls (cats that did not die) an increased risk of mortality was found for cats admitted to adoption centres unneutered, in poor health, and cats born at adoption centres. Cats aged 7 weeks or less and cats aged over 7 years were at an increased mortality risk compared to cats of other ages. Cats with disabilities (such as blindness) were at a higher mortality risk than cats without disabilities. A higher survival rate was found in a survey of cat shelters in Sweden where less than $10 \%$ of the relinquished cats were euthanized (Erikss on et al. 2009). Management optimizing the health of shelter cats and minimizing the introduction of infectious diseases in the face of a constantly changing population, potential overcrowding, and limited funds, remains a major challenge in shelter medicine (Litster et al. 2011). Knowledge of the risk factors can inform intervention strategies aimed at reducing the risk of cat mortality at adoption centres (Murray et al. 2008).

The Czech Republic is one of the countries where no healthy animals can be destroyed. The only legal justifications for euthanasia of stray animals are weakness, terminal illness, serious injury, genetic or congenital defect, overall exhaustion or old age of the animal, when survival would entail permanent suffering for the animal (Voslarova and Passantino 2012). The aim of the study was to analyze the records of the death and euthanasia rates of cats in Czech cat shelters in the period from 2011 to 2015.

\section{Materials and Methods}

The subjects of this retrospective study were cats impounded in three cat shelters situated in different regions of the Czech Republic. Out of a total of 2,170 cats, further analysis was performed on cats that died or had to be euthanized due to health reasons in the period from January 1, 2011, to December 31, 2015.

Male and female categories were used to determine sex. Based on the estimated or known ages, cats were divided into three age categories: cats younger than 6 months, cats aged 6 months to 50 months, and cats older than 50 months.

In order to determine the development and seasonal differences in cat deaths, the year of unassisted death or euthanasia (2011-2015), season (spring, summer, autumn, winter) and month of unassisted death or euthanasia were analyzed. In other words, numbers of cats that died or that were euthanized in individual years, seasons and months were determined. The numbers of cats that died or were euthanized in the shelters were also analyzed in relation to the time of death or euthanasia after admission.

Furthermore, the length of stay (LOS) of each cat was calculated. The LOS, in days, was the difference between the intake date and the date when the cat died or had to be euthanized due to health reasons.

The results were analyzed using the statistical package Unistat 5.6. (Unistat Ltd., London, England). Four independent variables were constructed from the shelter's original information regarding dead and euthanized cats: sex ( 2 levels: male, female) and age (3 levels: $<6$ months, 6-50 months, $>50$ months), year of death (5 levels: 2011, 2012, 2013, 2014, 2015), and season of death (4 levels: spring, summer, autumn, winter). First of all, the actual counts and relative frequencies (actual counts / total counts) of cats in all categories according to the monitored independent variables were calculated, and differences among these categories were tested. Actual and relative frequencies of dead and euthanized cats were determined also for individual months in the entire period of 2011-2015 and then mutually compared. Frequencies were compared on the basis of a chi-square analysis of $\mathrm{k} \times \mathrm{m}$ and $2 \times 2$ contingency tables (Zar 1999). In the process of LOS analysis, normality was checked 
using the Kolmogorov-Smirnov test (Zar 1999). As data were not distributed normally, non-parametric methods were used for testing. The effects of sex and age as independent variables on LOS as a dependent variable were analyzed. For LOS, the median was calculated for each level of the monitored independent variables. The effect of sex (2 level variable) was analyzed using two-tailed Mann-Whitney U test. The effect of age was analyzed by means of Kruskal-Wallis ANOVA and subsequently by non-parametric Tukey-type test using mean ranks (Zar 1999) as post hoc test for pairwise comparisons. A $P$-value $<0.05$ was considered as significant.

\section{Results}

In the period from 2011 to 2015,474 cats died and 240 had to be euthanized for health reasons. Altogether, death was the ultimate outcome for $33 \%$ of cats admitted in the shelters. Year and seasonal variations in the monitored period are given in Table 1. Death rates were consistent in individual years of the monitored period whereas significantly higher $(P<0.001)$ euthanasia rates were found in 2013 and 2014. Autumn and summer were the seasons with the majority of fatalities, $45.6 \%$ deaths and $33.8 \%$ euthanasias occurred in autumn and $34.6 \%$ deaths and $29.5 \%$ euthanasias occurred in summer.

Table 1. Year and seasonal variations in cat death and euthanasia rates.

\begin{tabular}{|c|c|c|c|c|}
\hline \multirow{2}{*}{ Variable } & \multicolumn{2}{|c|}{ Unassisted death } & \multicolumn{2}{|c|}{ Euthanasia } \\
\hline & No. & $\%$ & No. & $\%$ \\
\hline Year & 474 & $P>0.05^{\#}$ & 240 & $P<0.001^{\#}$ \\
\hline 2011 & 93 & $19.6^{\mathrm{a}}$ & 26 & $10.8^{\mathrm{b}}$ \\
\hline 2012 & 102 & $21.5^{\mathrm{a}}$ & 29 & $12.1^{\mathrm{b}}$ \\
\hline 2013 & 84 & $17.7^{\mathrm{a}}$ & 75 & $31.3^{\mathrm{a}}$ \\
\hline 2014 & 95 & $20.0^{\mathrm{a}}$ & 81 & $33.8^{\mathrm{a}}$ \\
\hline 2015 & 100 & $21.1^{\mathrm{a}}$ & 29 & $12.1^{\mathrm{b}}$ \\
\hline Season & 474 & $P<0.001^{\#}$ & 240 & $P<0.001^{\#}$ \\
\hline Spring & 35 & $7.4^{\mathrm{d}}$ & 46 & $19.2^{\mathrm{b}}$ \\
\hline Summer & 164 & $34.6^{\mathrm{b}}$ & 71 & $29.6^{\mathrm{a}, \mathrm{c}}$ \\
\hline Autumn & 216 & $45.6^{\mathrm{a}}$ & 81 & $33.8^{\mathrm{a}}$ \\
\hline Winter & 59 & $12.4^{\mathrm{c}}$ & 42 & $17.5^{\mathrm{b}, \mathrm{c}}$ \\
\hline
\end{tabular}

${ }^{a-c}-$ percentage and medians within a column and the same variable with different superscripts differ significantly $(P<0.05)$.

\# - Chi square test

Monthly variations in cat death and euthanasia rates are given in Fig. 1. The month with the highest death and euthanasia rates was October. In contrast, April was the month with the lowest death and euthanasia rates. Death and euthanasia rates were equal in individual months besides March when more cats were euthanized than died and September and November when more cats died than were euthanized.

The numbers of cats that died and were euthanized in the shelters in relation to the time after their admission are given in Table 2. Out of the cats that died, $319(67.3 \%)$ of them died within the first month and $95.4 \%$ of deaths occurred within 6 months after admission to the shelter. Similarly, $65.0 \%$ of all euthanized cats were euthanized within the first month and $88.3 \%$ of euthanasias were carried out within 6 months of admission.

Sex and age were recorded for $93.1 \%$ and $95.2 \%$ of the dead cats, respectively. The sex and age of dead cats and their median LOS are presented in Table 3. Significantly $(P<0.001)$ more females than males died or had to be euthanized in the shelters. In the cats that died, the median LOS of females was significantly $(P<0.001)$ shorter compared 


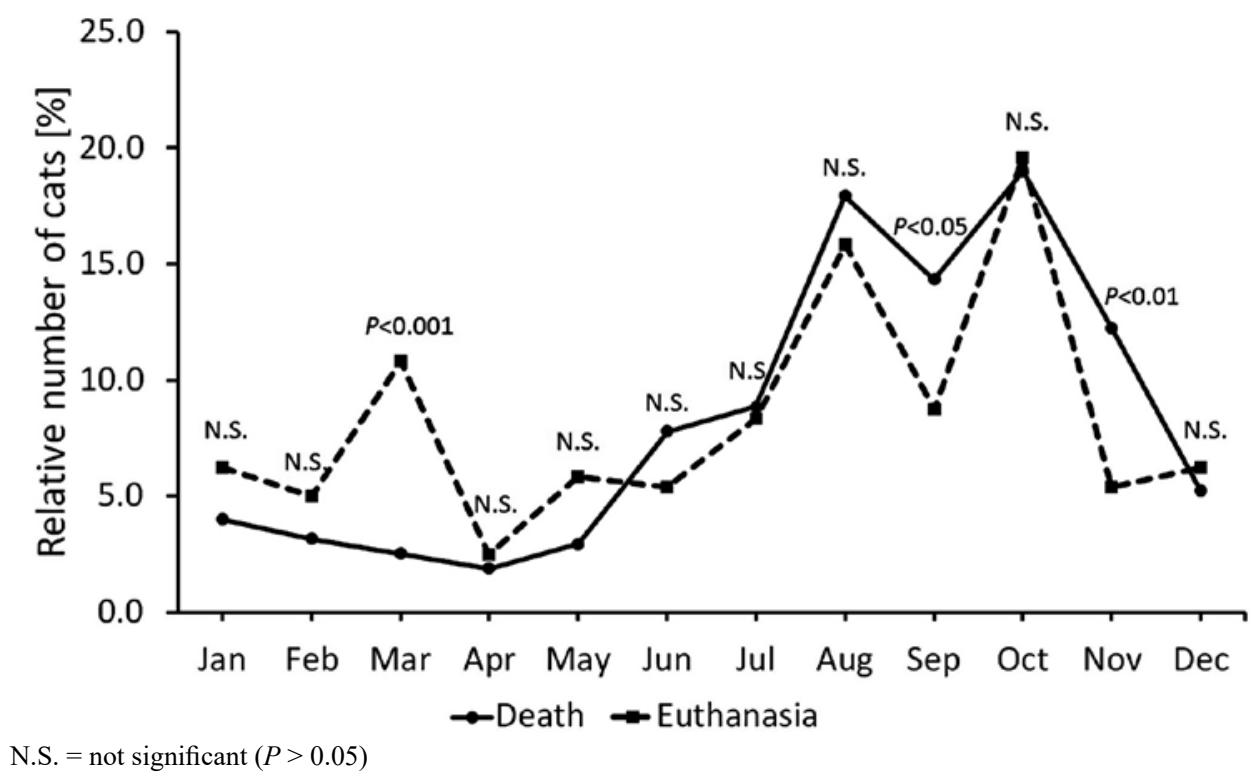

Fig. 1. Comparison of cats that died and cats that were euthanized in the shelters in individual months.

to the LOS of males. In euthanized cats, no difference was found between the LOS of males and females.

Among the dead cats, those younger than 6 months predominated and accounted for $73.7 \%$ of cats that died and $59.5 \%$ of euthanized cats. The shortest median LOS was found in cats older than 50 months. It was significantly shorter compared to the LOS of both younger categories.

\section{Discussion}

Ideally, no healthy, adoptable animal should be euthanized and most shelters strive to attain this goal. However, euthanasia will always be necessary for old and dying animals that are suffering (Bartlett et al. 2005). In the case of the shelters monitored in our study it is actually questionable whether euthanasia might have been applied more frequently in order to avoid prolonged suffering of cats before death. The number of cats that died was almost twice the number of cats that were euthanized. In a situation where the animal is seriously injured or ill with potentially reduced animal welfare, responsible shelter personnel or veterinarian need to consider treatment options or euthanasia. Euthanizing animals was established as a major stressor for personnel in animal shelters where euthanasia is performed on the basis of factors other than behaviour and health reasons (Rogelberg et al. 2007; Baran et al. 2009; Scotney et al. 2015). However, even if euthanasia was performed on medical grounds, concern over animal death was associated with reported traumatic stress. The mean level of stress reported by workers whose occupations required euthanizing animals did not vary across occupation, even though the reasons given for euthanasia did vary depending on context (Rohlf and Bennett 2005). Supposedly, it is not a popular decision even in cases of injured and ill animals and might be intentionally or subconsciously delayed by the responsible person. 
Table 2. Numbers of cats that died and cats that were euthanized in the shelters depending on the time after admission.

\begin{tabular}{lrrrr}
\hline \multirow{2}{*}{ Month } & \multicolumn{2}{c}{ Unassisted death } & \multicolumn{3}{c}{ Euthanasia } \\
\cline { 2 - 3 } \cline { 5 - 5 } & No. & $\%$ & No. & $\%$ \\
\hline 1 & 319 & 67.3 & 156 & 65.0 \\
2 & 67 & 14.1 & 14 & 5.8 \\
3 & 30 & 6.3 & 18 & 7.5 \\
4 & 22 & 4.6 & 11 & 4.6 \\
5 & 7 & 1.5 & 9 & 3.8 \\
6 & 7 & 1.5 & 4 & 1.7 \\
7 & 22 & 4.6 & 28 & 11.7 \\
Total & 474 & 100.0 & 240 & 100.0 \\
\hline
\end{tabular}

The health status of each cat should be assessed objectively, preferably by a veterinarian at the time of admission. Murray et al. (2008) found an increased risk of mortality in cats admitted to adoption centres in poor health. The medical records of 364 kittens taken into PAWS Chicago Adoption Centre in 2008 showed that only 104 (28.5\%) had no abnormalities identified in a physical examination performed by the shelter veterinarian at the time of intake (Litster et al. 2011). Our results suggest that a relatively high percentage of cats enter shelters in a very poor condition. Out of admitted cats, $21.9 \%$ were either euthanized or died within the first month of admission. Similarly, many animals were euthanized because they were in a condition of extreme suffering at admission to the zoonosis control centres in Votorantim. The mean survival time of animals that were euthanized was 20 days, and the median was 9 days from the date of capture to the date of euthanasia (Ardila et al. 2015). The other most frequent fates of animals captured were death due to injury or disease. In our study, the death rates were more or less double the euthanasia rates in the first four months after admission. Again, it might be the result of attempts to cure animals that should have been euthanized earlier instead. In such cases euthanasia should be considered as a humane relief of suffering in contrast with the prolonged suffering of animals that are kept alive in spite of their incurable health problems.

The majority of deaths and euthanasias occurred within four months after admission. Out of all fatalities, only $7.6 \%$ cats died and $17.1 \%$ were euthanized more than four months

Table 3. Number of cats that died and cats that were euthanized in the shelters and their length of stay (LOS) by sex and age.

\begin{tabular}{|c|c|c|c|c|c|c|}
\hline \multirow[b]{2}{*}{ Variable } & \multicolumn{3}{|c|}{ Unassisted death $(\mathrm{n}=474)$} & \multicolumn{3}{|c|}{ Euthanasia $(n=240)$} \\
\hline & No. & $\%$ & $\begin{array}{c}\text { LOS } \\
\text { (Median of days) }\end{array}$ & No. & $\%$ & $\begin{array}{c}\text { LOS } \\
\text { (Median of days) }\end{array}$ \\
\hline Sex & 444 & $P<0.001^{\#}$ & $P<0.001^{\&}$ & 221 & $P=0.001^{\#}$ & $P>0.05^{\&}$ \\
\hline Male & 173 & $39.0^{\mathrm{b}}$ & $23.0^{\mathrm{a} 93}$ & $42.1^{\mathrm{b}}$ & $14.0^{\mathrm{a}}$ & \\
\hline Female & 271 & $61.0^{\mathrm{a}}$ & $16.0^{\mathrm{b}}$ & 128 & $57.9^{\mathrm{a}}$ & $19.0^{\mathrm{a}}$ \\
\hline Age & 460 & $P<0.001^{\#}$ & $P=0.019^{\&}$ & 220 & $P<0.001^{\#}$ & $P<0.001^{\&}$ \\
\hline$<6$ months & 339 & $73.7^{\mathrm{a}}$ & $17.0^{\mathrm{a}}$ & 131 & $59.5^{\mathrm{a}}$ & $16.0^{\mathrm{a}}$ \\
\hline $6-50$ months & 65 & $14.1^{\mathrm{b}}$ & $28.0^{\mathrm{a}}$ & 61 & $27.7^{\mathrm{b}}$ & $26.0^{\mathrm{a}}$ \\
\hline$>50$ months & 56 & $12.2^{\mathrm{b}}$ & $14.5^{\mathrm{b}}$ & 28 & $12.7^{\mathrm{c}}$ & $0.0^{\mathrm{b}}$ \\
\hline
\end{tabular}

a-c - percentage and medians within a column and the same variable with different superscripts differ significantly $(P<0.05)$.

\# - Chi square test

\&- Kruskal-Wallis ANOVA (Mann-Whitney test for sex) 
after admission. Our results are in agreement with the results of a study analyzing the risk of mortality in cats kept at the adoption centres of a UK cat charity. Murray et al. (2008) reported that the risk of mortality decreased as the time in the adoption centre increased. The increasing risk of contracting an infection for cats staying longer in shelters found in other studies (Pedersen et al. 2004; Dinnage et al. 2009) did not seem to affect the mortality rate in the shelters monitored in our study. It can be assumed that the shelters in our study adopted effective methods of reducing the exposure of cats to infectious diseases. It is virtually impossible to prevent the entry of infectious diseases into rescue facilities and shelters; hence it is essential that buildings and management practices are designed so as to limit their spreading (Sturgess 2007).

Monthly and seasonal variations in mortality rates reflect the seasonality of cat admissions. The increase in the intake of kittens following the feline breeding season (Rinzin et al. 2008; Alberthsen et al. 2016) is likely to be responsible for the increase in death and mortality rates in shelter cats in summer and autumn. It is in agreement with our findings that most deaths and euthanasias occurred shortly after admission.

Significantly more females than males died or had to be euthanized in the shelters. Even when the common overrepresentation of female cats in shelters (Lepper et al. 2002; Wallace and Levy 2006; Alberthsen et al. 2016) is considered, the female to male sex ratio among dead cats was greater in comparison with the sex ratio of cats admitted to the monitored shelters. Considering the mortality rate and median LOS, our results suggest that females are admitted to shelters in a worse condition than males and that males have a higher survival rate.

Death and euthanasia rates also varied with the age of cats. Cats younger than 6 months accounted for $73.7 \%$ of the cats that died and $59.5 \%$ of euthanized cats. Compared to cat admissions, more than one third of the cats admitted to the monitored shelters at an age of less than 6 months died or had to be euthanized. A high death rate is more likely to be expected in feral kittens (Levy et al. 2003) rather than in those under intensive human care. Further research is needed to establish what reasons are responsible for such high mortality rates in kittens and juvenile cats in shelters. Besides poor condition at the time of admission and an inability to raise orphaned neonatal kittens it might be also due to an increased susceptibility to infectious diseases in young cats, whose immune system is not yet fully developed. Murray et al. (2008) reported that not only cats aged 7 weeks or less and cats born at adoption centres, but also cats aged over 7 years are at an increased mortality risk compared to cats of other ages. In our study, the death and euthanasia of cats older than 50 months was not so frequent, however, their median LOS was significantly shorter in comparison to the LOS of both younger categories. The median time to euthanasia of cats older than 50 months ( 0 days) may mean that at intake, the persons responsible were more ready to euthanize older cats than younger ones.

In conclusion, $33 \%$ of the cats admitted to the shelters monitored in our study died or had to be euthanized for health reasons. It is within the range of published death rates in comparable shelters varying from less than 10\% in Sweden (Eriksson et al. 2009) to $40 \%$ in Brazil (Ardila Galvis et al. 2015). A limitation of this study as well as other shelter studies is the lack of medical records but most shelters do not have the resources for diagnostic testing nor post mortem examination. However, since the majority of mortalities occurred shortly after admission, it is more likely that they resulted from the poor condition of the cats at the time of admission than from the shelter's environment. Females and cats younger than 6 months had an increased risk of mortality. In autumn and summer, shelter operators face increased death and euthanasia rates and supposedly also related costs for the treatment of cats prior to death. In general, the number of cats that died was almost double the number of cats that were euthanized suggesting that attempts to cure cats were preferred over euthanasia even in cases of serious health problems. 


\section{References}

Alberthsen C, Rand JS, Bennett PC, Paterson M., Lawrie M, Morton JM 2013. Cat admissions to RSPCA shelters in Queensland, Australia: description of cats and risk factors for euthanasia after entry. Aust Vet J 91: 35-42

Alberthsen C, Rand J, Morton, J, Bennett P, Paterson M, Vankan D 2016: Numbers and characteristics of cats admitted to Royal Society for the Prevention of Cruelty to Animals (RSPCA) shelters in Australia and reasons for surrender. Animals 6: 3

Ardila Galvis, JO, Baquero OS, Dias RA, Ferreira F, Chiozzotto EN, Hildebrand Grisi-Filho JH 2015: Monitoring techniques in the capture and adoption of dogs and cats. Geospat Health 10: 158-162

Arkow P 1994: A new look at pet overpopulation. Anthrozoos 7: 202-205

Baran BE, Allen JA, Rogelberg SG, Spitzmuller C, DiGiacomo NA, Webb JB, Carter NT, Clark OL, Teeter LA, Walker AG 2009: Euthanasia-related strain and coping strategies in animal shelter employees. JAVMA-J Am Vet Med A 235: 83-88

Bartlett PC, Bartlett A, Walshaw S, Halstead S 2005: Rates of euthanasia and adoption for dogs and cats in Michigan animal shelters. J Appl Anim Welf Sci 8: 97-104

Carter CN 1990: Pet population control - another decade without solutions. JAVMA-J Am Vet Med A 197: $192-195$

Cave TA, Thompson H, Reid SWJ, Hodgson DR, Addie DD 2002: Kitten mortality in the United Kingdom: a retrospective analysis of 274 histopathological examinations (1986 to 2000). Vet Rec 151: 497-501

Dinnage JD, Scarlett JM, Richards JR 2009: Descriptive epidemiology of feline upper respiratory tract disease in an animal shelter. J Feline Med Surg 11: 816-825

Eriksson P, Loberg J, Andersson M 2009: A survey of cat shelters in Sweden. Anim Welfare 18: 283-288

Lepper M, Kass PH, Hart LA 2002: Prediction of adoption versus euthanasia among dogs and cats in a California animal shelter. J Appl Anim Welf Sci 5: 29-42

Levy JK, Gale DW, Gale LA 2003: Evaluation of the effect of a long-term trap-neuter-return and adoption program on a free-roaming cat population. JAVMA-J Am Vet Med A 222: $42-46$

Litster A, Allen J, Mohamed A, He S 2011: Risk factors for delays between intake and veterinary approval for adoption on medical grounds in shelter puppies and kittens. Prev Vet Med 101: 107-112

Murray JK, Skillings E, Gruffydd-Jones TJ 2008: A study of risk factors for cat mortality in adoption centres of a UK cat charity. J Feline Med Surg 10: 338-345

Nassar R, Mosier JE, Williams LW 1984: Study of the feline and canine populations in the greater Las Vegas area. Am J Vet Res 45: 282-287

Onodera N, Uchida K, Kakuma Y 2014: Association between characteristics of cats and satisfaction of owners who adopted cats from an animal hospital in Japan. J Vet Med Sci 76: 729-733

Patronek GJ, Glickman LT, Beck AM, McCabe GP, Ecker C 1996: Risk factors for relinquishment of cats to an animal shelter. JAVMA-J Am Vet Med A 209: 582-588

Pedersen NC, Sato R, Foley JE, Poland AM 2004: Common virus infections in cats, before and after being placed in shelters, with emphasis on feline enteric coronavirus. J Feline Med Surg 6: 83-88

Rinzin K, Stevenson MA, Probert DW, Bird RG, Jackson R, French NP, Weir JA 2008: Free-roaming and surrendered dogs and cats submitted to a humane shelter in Wellington, New Zealand, 1999-2006. N Z Vet J 56: 297-303

Rogelberg SG, Reeve CL, Spitzmuller C, DiGiacomo N, Clark OL, Teeter L, Walker AG, Starling PG, Carter NT 2007: Impact of euthanasia rates, euthanasia practices, and human resource practices on employee turnover in animal shelters. JAVMA-J Am Vet Med A 230: 713-719

Rohlf V, Bennett P 2005: Perpetration-induced traumatic stress in persons who euthanize nonhuman animals in surgeries, animal shelters, and laboratories. Soc Anim 13: 201-219

Rowan AN 1992: Shelters and pet overpopulation - a statistical black-hole. Anthrozoos 5: 140-143

Scotney RL, McLaughlin D, Keates HL 2015: A systematic review of the effects of euthanasia and occupational stress in personnel working with animals in animal shelters, veterinary clinics, and biomedical research facilities. JAVMA-J Am Vet Med A 247: 1121-1130

Sturgess K 2007: Disease and welfare. In: Rochlitz I (Ed.): The Welfare of Cats. Springer, Dordrecht, pp. 205-225

Voslarova E, Passantino A 2012. Stray dog and cat laws and enforcement in Czech Republic and in Italy. Ann Ist Super Sanita 48: 97-104

Wallace JL, Levy JK 2006: Population characteristics of feral cats admitted to seven trap-neuter-return programs in the United States. J Feline Med Surg 8: 279-284

Zar JH 1999: Biostatistical Analysis. $4^{\text {th }}$ edn. Prentice-Hall, Inc., New Jersey, 663 p. 